\title{
Neurotrophic keratopathy — case reports analysis and management
}

\author{
Ioannis Mallias, Panagiota Mylova, Anastasia Tassiopoulou
}

Laser Plus Eye, Nea Smyrni, Athens, Greece

\begin{abstract}
This is a case series of patients suffering from neurotrophic keratopathy. The main categorisation of the disease is discussed as well as the management that should be followed on each stage. Several cases of patients with neurotrophic keratopathy are analysed and the treatment (different on every patient) of them is presented. Newer substances that are beginning to be used in the treatment of neurotrophic keratopathy are discussed as well.
\end{abstract}

KEY WORDS: neurotrophic keratopathy, cornea, corneal perforation, corneal ulcers, herpetic keratitis, penetrating keratoplasty, neurotrophic ulcer

Ophthalmol J 2017; Vol. 2, No. 3, 91-99

\section{INTRODUCTION}

Neurotrophic keratopathy is a degenerative disease that is characterised by reduction of corneal sensitivity. In neurotrophic keratopathy, the corneal innervation that is procured to the cornea by the trigeminal nerve is impaired. Neurotrophic keratopathy is estimated to affect less than 50/100,000 individuals [1].

Every condition, ocular or systemic, that alters corneal sensory innervation (from the cornea itself to the trigeminal nerve) can cause neurotrophic keratopathy. Some of the most common conditions that can lead to neurotrophic keratopathy are herpes keratitis (simplex and zoster), contact lens overuse, topical anaesthetic abuse, chemical and physical burns, topical drug toxicity, and corneal and ocular surgery.

When it comes to corneal surgery, there have been studies $[2,3]$ indicating that both Photorefractive Keratectomy (PRK) and laser in situ keratomileusis (LASIK) can potentially cause nerve damage. However, the chances of something like this happening are higher $[4,5]$ in LASIK surgery. Deep anterior lamellar keratoplasty [6] (DALK) and penetrating keratoplasty $(\mathrm{PK})$ can also cause a degree of corneal insensitivity. In patients with keratoconus [7], who have undergone corneal collagen crosslinking, a reduction of corneal sensitivity occurs as well.

Laser for the treatment of proliferative diabetic retinopathy has also been reported [8] to cause neurotrophic keratopathy. Vitrectomy (for retinal detachment) and photocoagulation to treat diabetic retinopathy have been reported [9] to cause neurotrophic keratopathy, as well. Also, neurosurgical procedures [10] or trauma damaging the fifth cranial nerve, intracranial tumours, multiple sclerosis, aneurisms, and stroke can cause neurotrophic keratitis. Diabetes, vitamin A deficiency, and leprosy have also been linked [10] to neurotrophic keratopathy.

During clinical examination, many histological alterations may be observed. Thinning or disruption of the epithelial layer, swelling of the epithelial cells, disorganisation of Bowman's membrane, stromal melting, or scarring and neovascularisation are several of them. Conjunctiva is usually also involved with the reduction in the density of goblet cells. 
The highly concerning thing about neurotrophic keratopathy is that patients usually do not seek medical opinion, due to the lack of sensitivity on the cornea, thus the lack of ocular surface symptoms, and the disease progresses until they do, months or maybe years after its first manifestation.

Starting the clinical evaluation, the physician should evaluate corneal sensitivity, when neurotrophic keratopathy is suspected. Subsequently, corneal staining with fluorescein should be performed in order to check for epithelial deficits. A Schirmer test could also be useful in order to determine whether the tear production has been compromised due to reduction of corneal sensitivity. Finally, in order to rule out any chance of infection, corneal culture should be performed.

Based on its symptoms, neurotrophic keratopathy can be categorised in three stages, known as the "Mackie classification" [11]:

Stage 1: this stage is manifested by hyperplasia or irregularity of the corneal epithelium that can evolve to punctate keratopathy, corneal oedema, stromal scarring, and neovascularisation.

Stage 2: this stage is presented with recurrent and persistent epithelial defects, most commonly in the superior half of the cornea. These defects are usually oval-shaped and their margins are smooth and rolled due to the impaired epithelial healing.

Stage 3: in this stage, there is involvement of the stroma, which leads to corneal ulcer, melting, and corneal perforation.

When managing neurotrophic keratopathy, the main purpose is to promote corneal healing without any complications. Topical non-steroidal anti-inflammatory drugs (NSAIDs) [12] should be avoided because they do not show any improvement in patients with neurotrophic keratopathy and they can further decrease the sensitivity of the cornea. The management in stage 1 of the disease, where punctate keratopathy is clinically observed, is with frequent application of preservative-free artificial tears and lubricant ointments. The main purpose is to improve the quality of the epithelium and avoid epithelial breakdown. In cases where punctate keratopathy is persistent, autologous serum $[13,14]$ can be used, as well as a therapeutic soft contact lens.

In stage 2 , the main purpose of management is to heal the persistent epithelial defects and prevent the formation of corneal ulcers. The treatment usually includes plenty of artificial tears and lubricant ointments, autologous serum use, amniotic membrane [14] graft, or tarsorrhaphy, if needed, in order to manage the epithelial defects. Because the epithelium is open, antibiotic drops can be prescribed in order to prevent infections [1].

In stage 3, the treatment is focused on the prevention of corneal perforation. In addition to the treatment used for stages 1 and 2, in stage 3 oral doxycycline, medroxyprogesterone, can be prescribed if stromal melting is observed [15]. A ReGeneraTing Agent (RGTA) polymer [16] eye drop has also proven to be successful in treating persistent neurotrophic ulcers. Similarly, a recombinant version of human nerve growth factor (Cenegermin) [17] has been proven to restore the normal healing process of the eye and repair corneal damage for patients with moderate to severe neurotrophic keratopathy and has recently been granted approval for use in Europe [17].

If, despite all efforts, perforation of the cornea occurs, the treatment varies and depends on its size. If it is small, cyanoacrylate glue can be applied, along with a soft contact lens. If the perforation is larger, a conjunctival flap procedure can be performed or even a lamellar keratoplasty, depending on the severity of the case.

\section{CASE REPORTS}

These are the case reports, presenting several different patients with neurotrophic keratitis and the pattern we chose to treat them.

\section{PATIENT 1}

The first patient was male, 66 years old. He was diagnosed with Sjögren's syndrome and severe acne rosacea that intensified the severe blepharitis he already had. The patient had a history of recurrent neurotrophic ulcers in both eyes. The patient was thoroughly examined by Dr Mallias (with slit lamp and anterior optical coherence tomography [OCT] as shown in Figures 1 and 2). He was diagnosed with keratoconjunctivitis sicca on the right eye and a sterile corneal ulcer on the left eye (Fig. 1), and his best corrected visual acuity (BCVA) was 20/30 in the right eye and 20/200 in the left eye.

The patient was treated with ample artificial tears and an antibiotic (preventively), and a contact lens was placed. After three days the patient felt slightly better and the contact lens was removed, but he returned a few days later complaining of pain and severe loss of visual acuity. The slit lamp examination revealed corneal perforation and reflux of blood of Schlemm's canal due to hypotony (Fig. 2). 


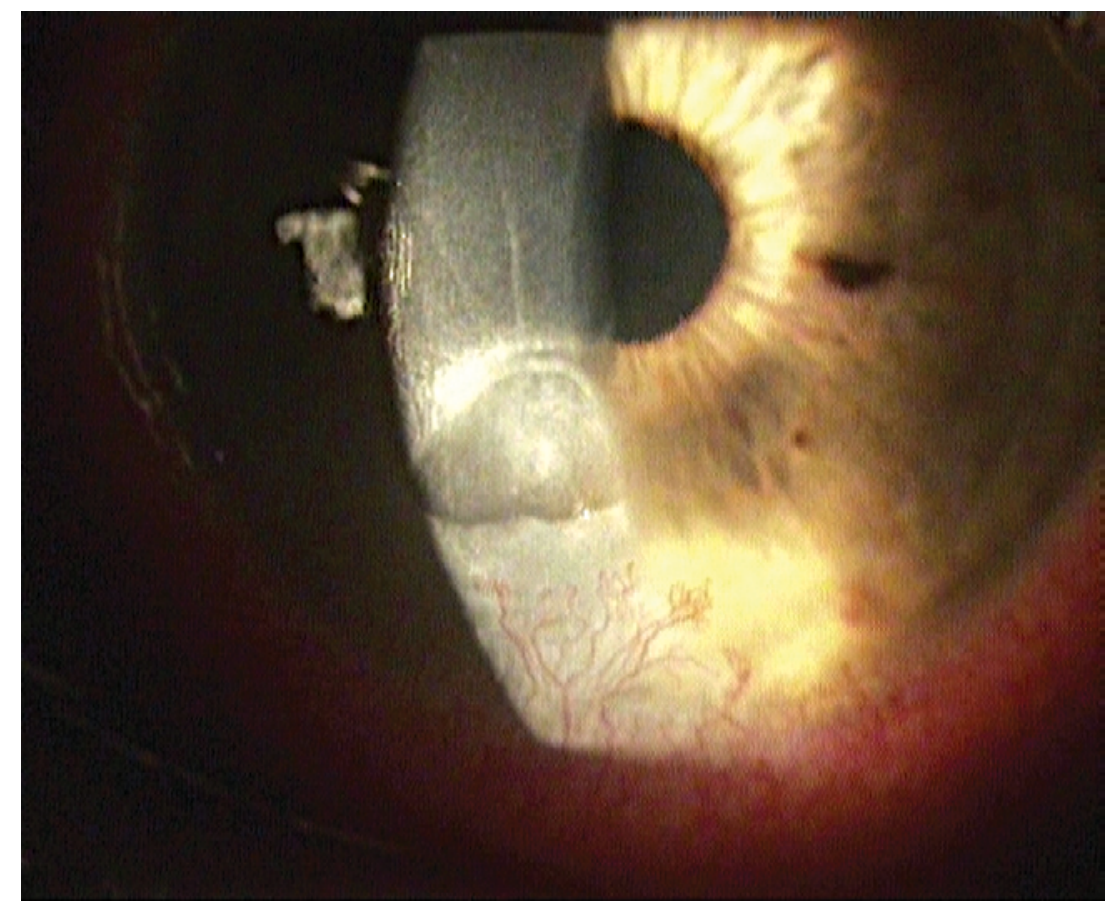

FIGURE 1. Slit lamp photo from the ulcer of the left eye of the first patient

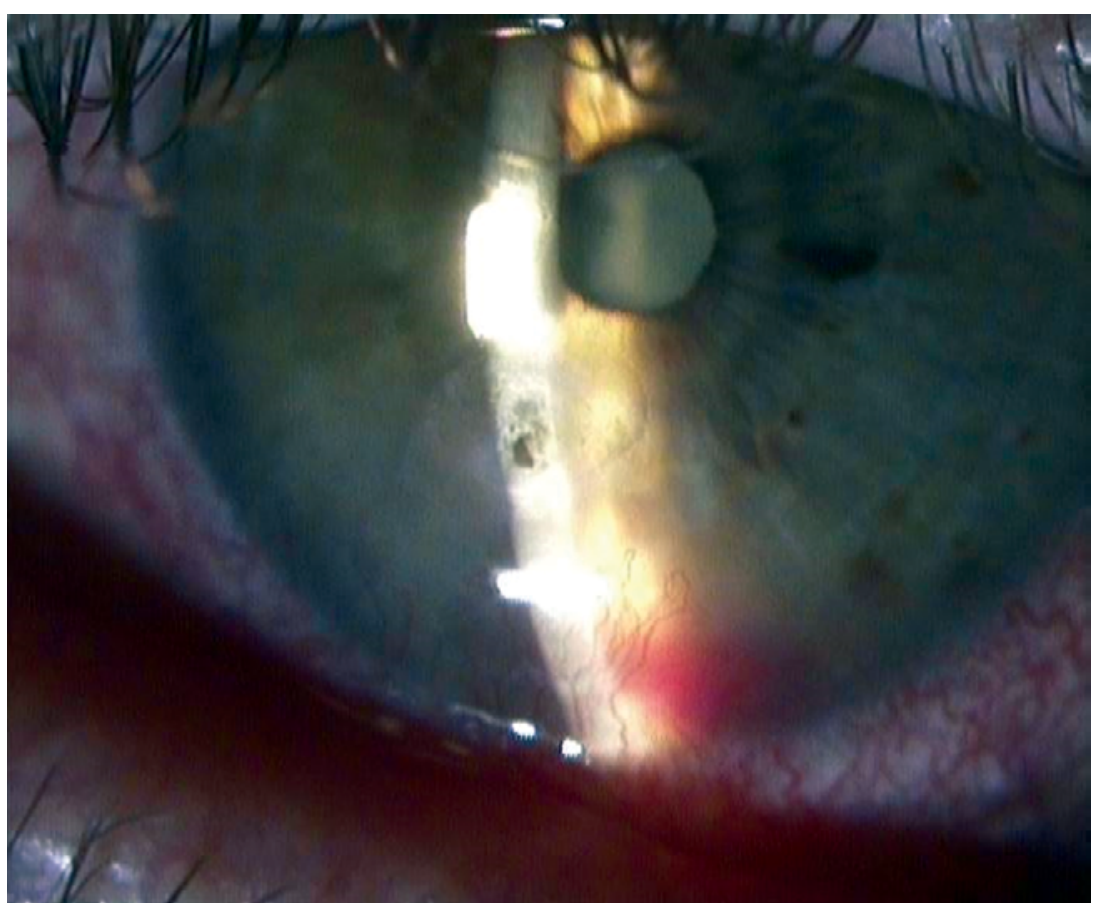

FIGURE 2. The left eye of the first patient with corneal perforation

Dr Mallias then proceeded with a conjunctival flap procedure and, after two weeks, a successful penetrating keratoplasty procedure (Fig. 3).

\section{PATIENT 2}

The second patient was female, 64 years old, diagnosed with biopsy-proven ocular cicatricial pem- 


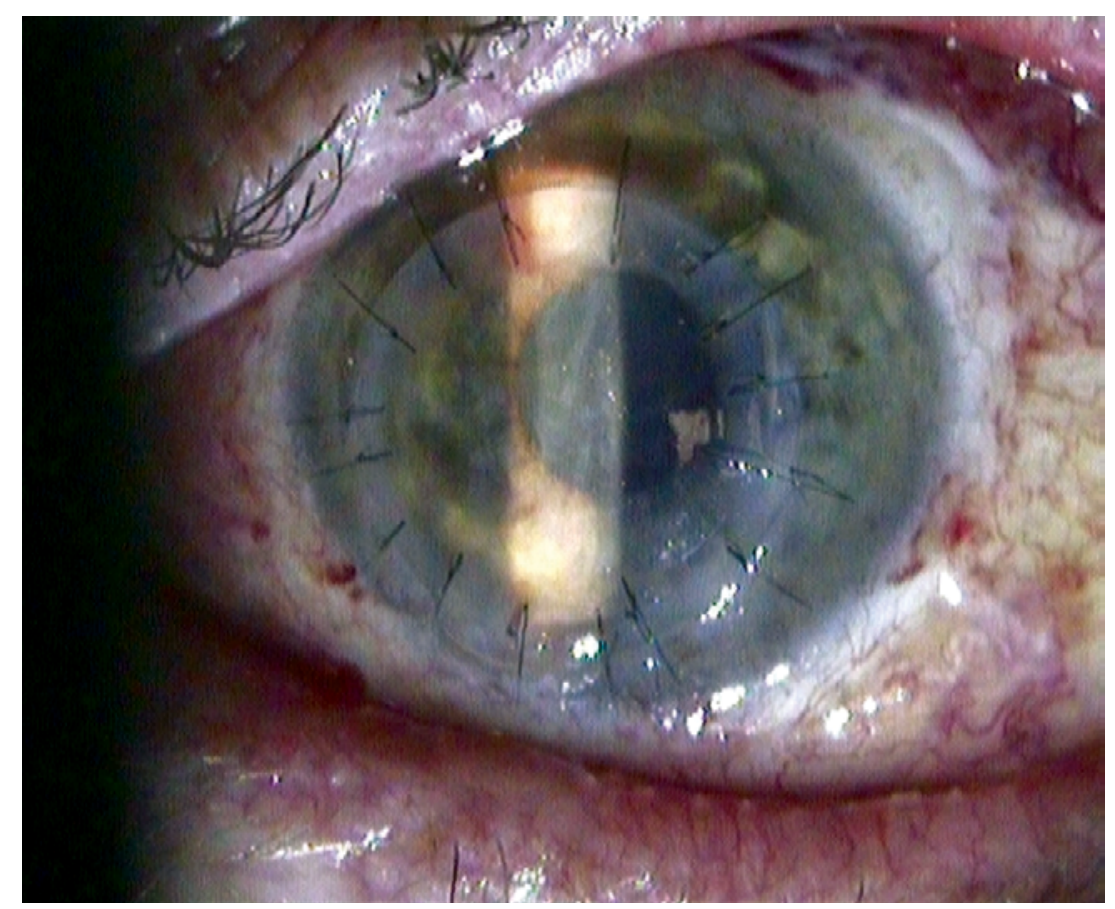

FIGURE 3. The left eye of the first patient after the penetrating keratoplasty

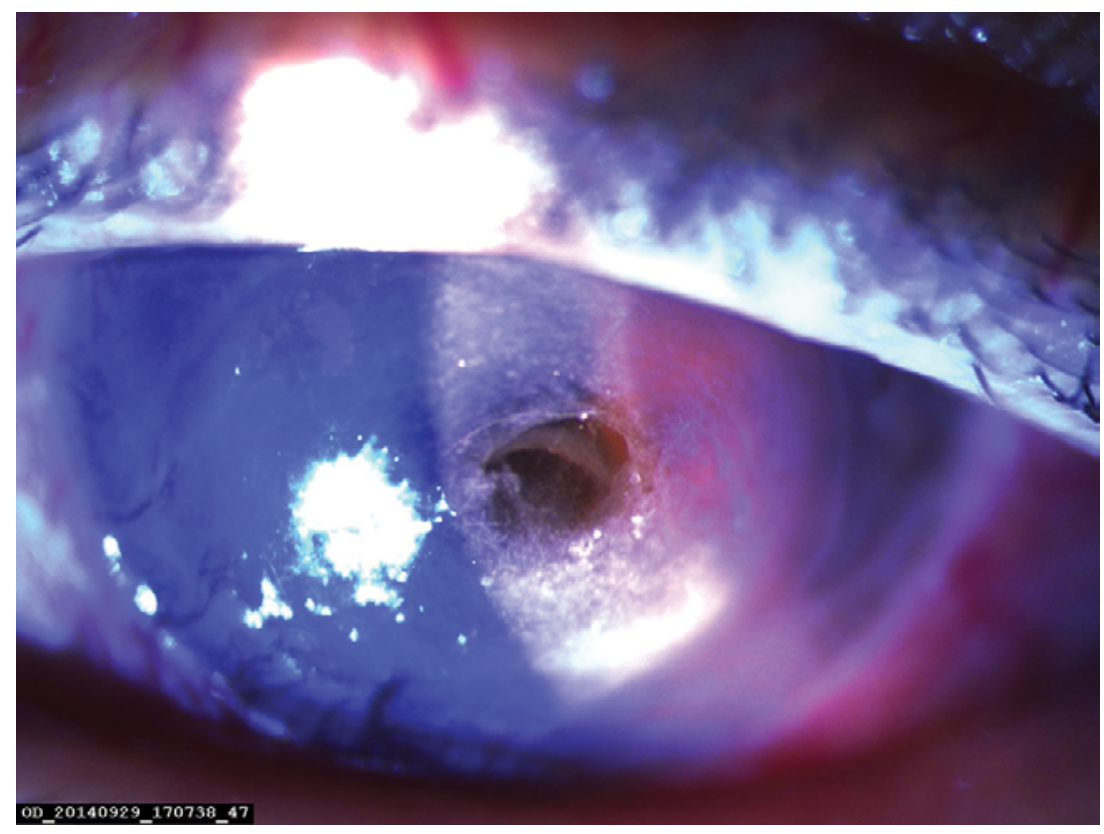

FIGURE 4. The left eye of the second patient. A large epithelial defect is observed. There is also a large area of opacification of the cornea, and in the centre there is a small area of descemetocele through which she was able to see $20 / 200$

phigoid. Her BCVA was 20/32 in the right eye and 20/200 in the left eye (Fig. 4).

She underwent an emergency penetrating keratoplasty (Fig. 5) of the left eye due to eminent risk of corneal perforation.

The patient developed a non-healing epithelial defect five months after penetrating keratoplasty in her left eye (Fig. 6). Artificial tears and a bandage soft contact lens were applied, and oral doxycycline was prescribed.

Despite the treatment the epithelial defect expanded and covered more than half of the corneal surface (Fig. 7). Cortical steroids were discontinued and oral valacyclovir was initiated at $500 \mathrm{mg}$ twice a day. 


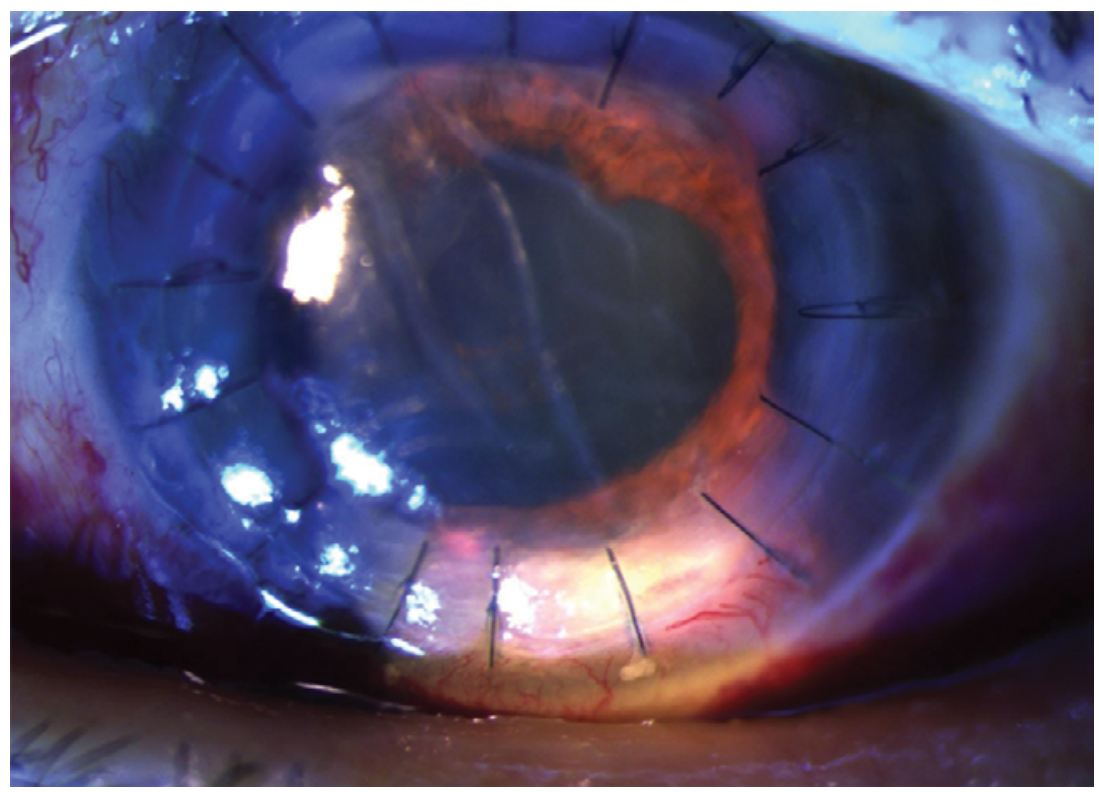

FIGURE 5. First postoperative day after a penetrating keratoplasty in Patient 2. There is still mild corneal oedema but good graft apposition to the host cornea with 16 interrupted sutures
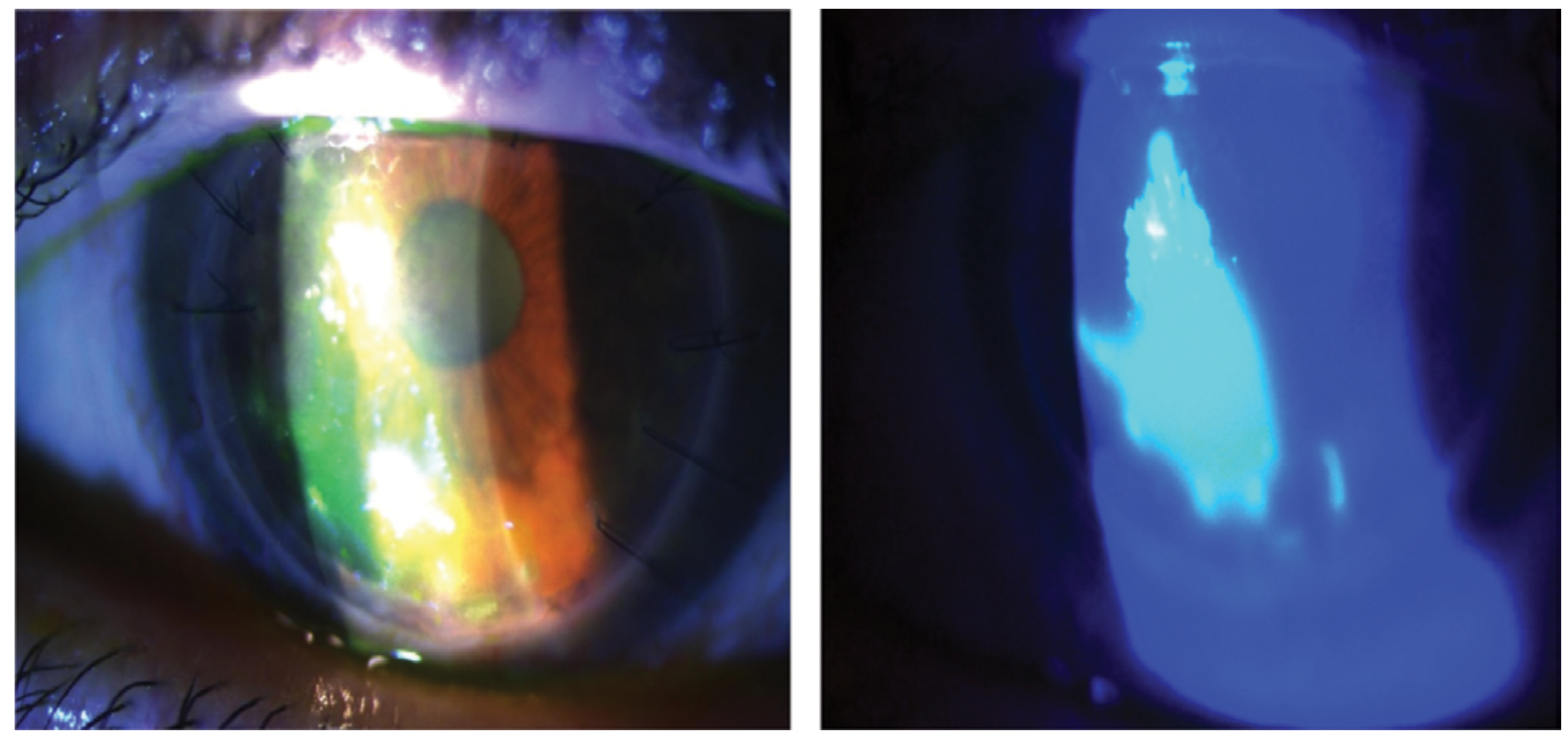

FIGURE 6. Epithelial defect over the corneal graft five months after the penetrating keratoplasty. Right image shows the epithelial defect stained with fluorescein. Left image shows the defect with cobalt blue light

Despite the change of medical treatment, epithelialisation was not achieved. At that point, the decision to start the patient on RGTA polymer eye drop was made. The dosage was one drop every other day. Several days after treatment initiation, the epithelium healed and the patient's visual acuity improved significantly (Fig. 8).

\section{PATIENT 3}

The third patient was female, 87 years old. She complained about lacrimation and severe pain in her right eye. Her BCVA was 20/200 for the right eye and 20/25 for the left eye. Slit lamp and OCT examination revealed corneal melt with a small descemetocele (area of the cornea with total melt of 


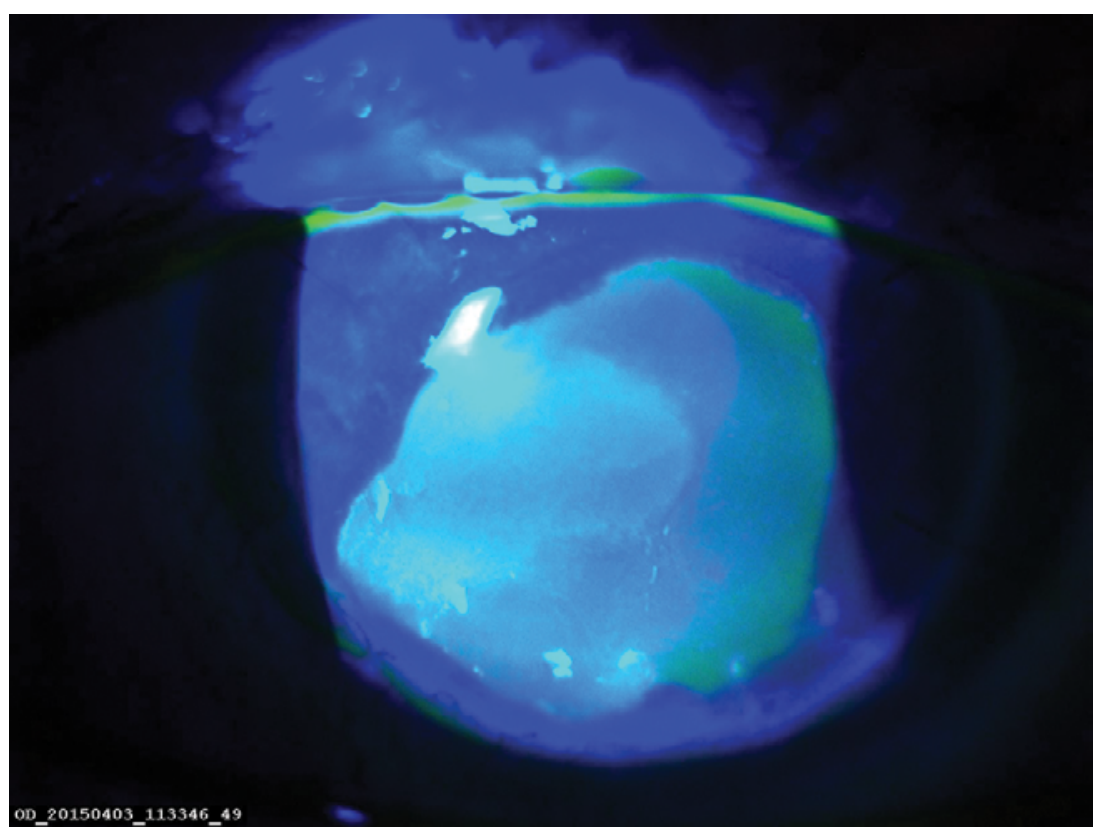

FIGURE 7. Expanded epithelial defect after treatment initiation
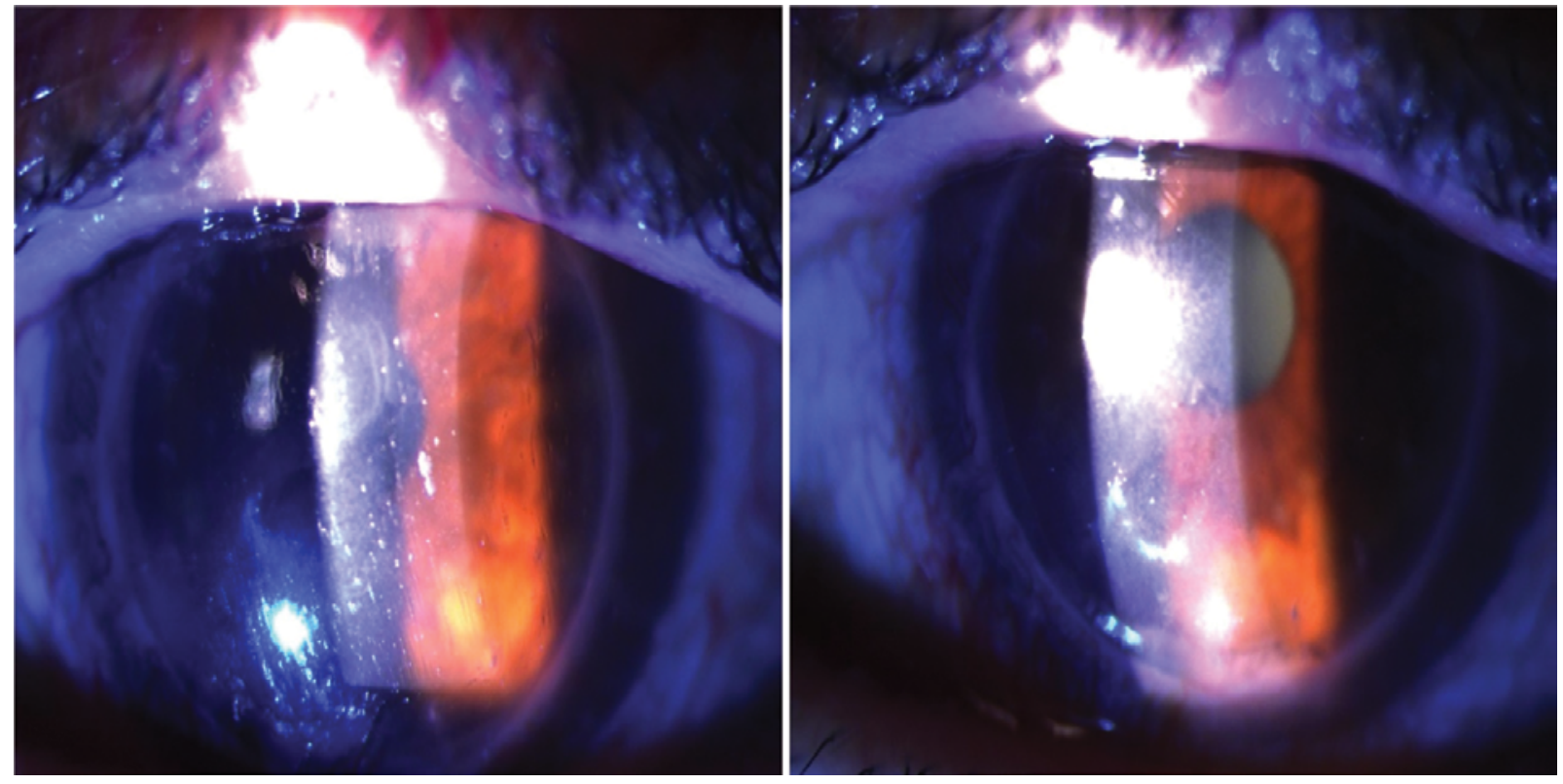

FIGURE 8. A gradual decrease of the epithelial defect is observed after the initiation of treatment with RGTA polymer drops

the corneal stroma where only Descemet's membrane is intact) on the right eye (Figs. 9 and 10).

Dr Mallias then proceeded to treat with Histoacryl glue (Figs. 11, 12) and a bandage soft contact lens. The patient was instructed to instil ofloxacin four times per day and preservative free artificial tears every two hours. The use of topical corticosteroids is contraindicated in such cases.
The Histoacryl glue remains on the cornea until healing is achieved. Then the glue usually falls out by itself

\section{DISCUSSION}

Neurotrophic keratopathy is a complicated disease and should be treated carefully. Patients should be 


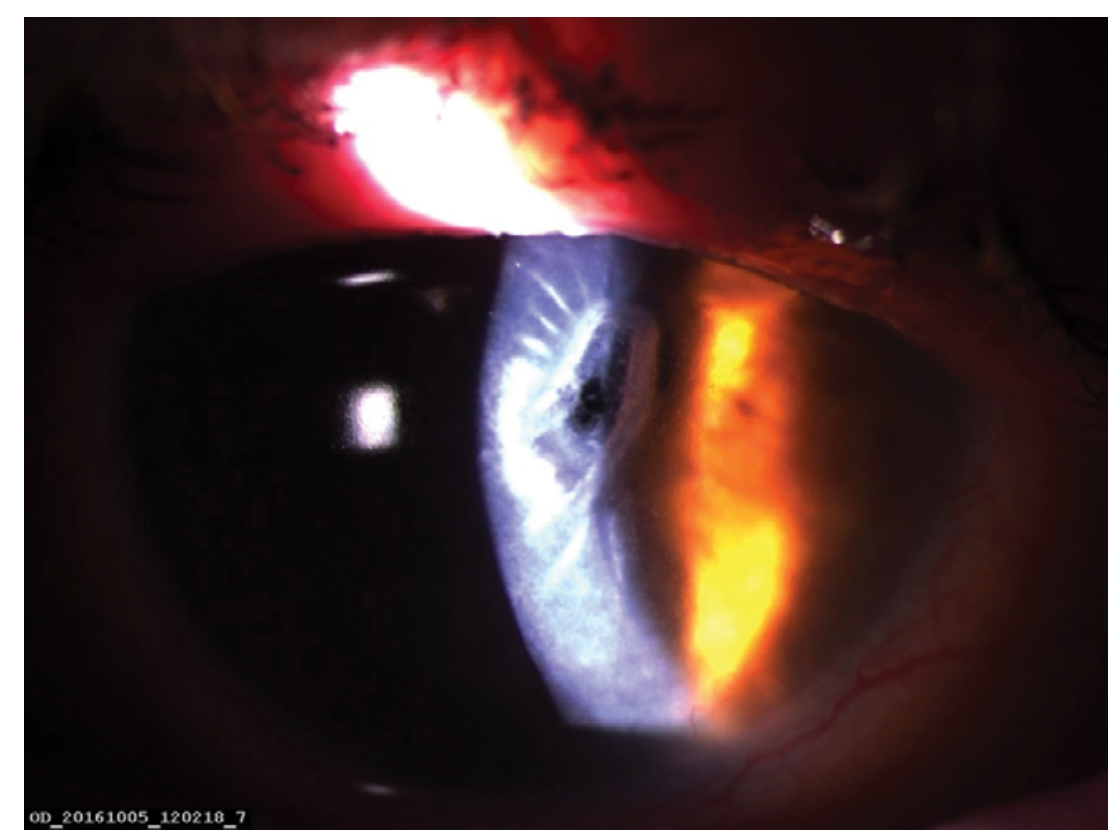

FIGURE 9. Corneal melt with a small descemetocele in the centre

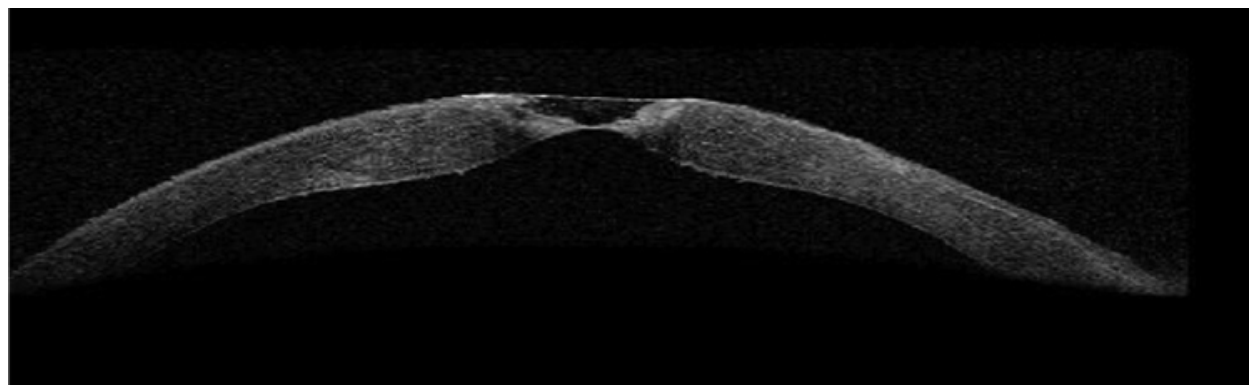

FIGURE 10. OCT image of the right eye of the third patient before application of Histoacryl glue

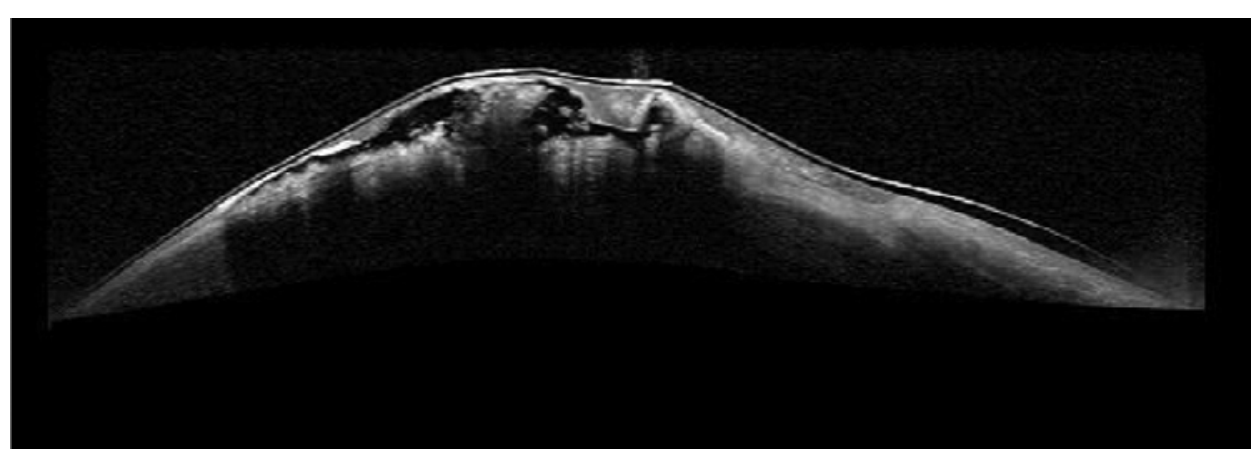

FIGURE 11. OCT image after the Histoacryl glue placement

closely monitored due to the lack of corneal sensitivity. In order to make the diagnosis of neurotrophic keratopathy, corneal sensation should be evaluated. To test for corneal sensation there are qualitative and quantitative methods [18]. The most commonly used method in clinical practice, which is qualitative in nature, is the use of a cotton-tipped applicator. A wisp of the cotton-tipped applicator is used to compare sensation in each eye. It is recommended to approach the patient from the side and test all four quadrants. The 


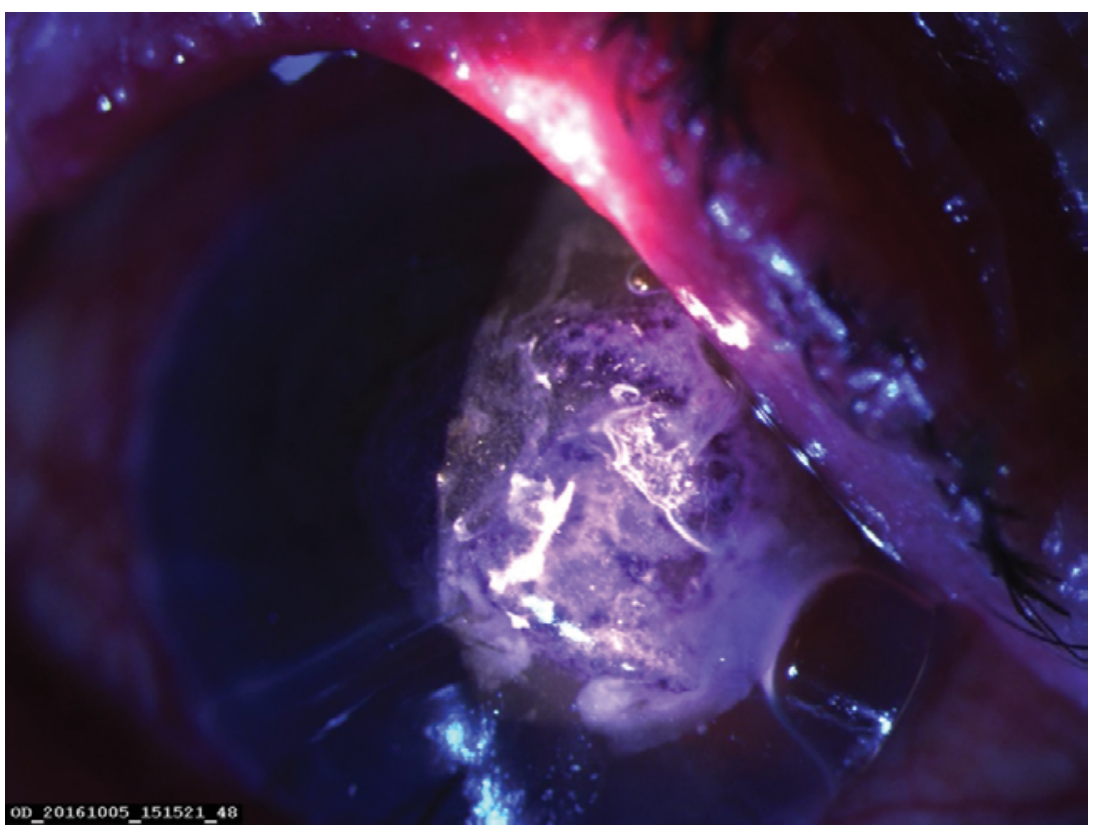

FIGURE 12. The right eye of the third patient after the Histoacryl glue placement

sensation should be recorded in each location as: normal, reduced, or absent. Topical anaesthetics should not be used prior to testing corneal sensation. Corneal esthesiometers could be used as well, such as the Handheld Cochet-Bonnet esthesiometer [18].

Artificial tears should be used frequently in order to smooth out the cornea in all the stages of the disease, and patients should be advised to be extra careful to avoid infections because the corneal epithelium might be compromised. Even after treating the current symptoms, patients should be extra careful and pay frequent visits to the ophthalmologist in order to make sure that the ocular surface remains healed.

Due to the nature of the disease, it is difficult to manage it with drugs because the preservatives that exist in them cause more harm than good on the ocular surface. This is the reason why scientists came up with the ReGeneraTing Agent (RGTA) eye drop and the recombinant version of human nerve growth factor (Cenegermin). Both of these substances promote epithelial healing.

RGTA [19] acts by playing the role of glycosaminoglycan, more specifically, heparin sulphate. The absence of glycosaminoglycan is associated with degradation of the extracellular matrix, which can result in corneal thinning, non-healing epithelial defects, and inflammation. The RGTA agents restore the balance of matrix proteins and cytokines that are normally bound to heparin sulphate and ensure equilibrium in the corneal microenvironment. This prevents degradation of extracellular matrix proteins, and promotes stromal and, subsequently, epithelial healing. Cenegermin [17] or recombinant human nerve growth factor as mentioned above is an artificial form of a naturally occurring signalling protein, nerve growth factor (NGF). It aims to improve nerve function in the cornea and stimulate healing as well as bring back some of the lost sensation of the cornea. RGTA agents have been further investigated and used in dealing with neurotrophic keratopathy. On the other hand, Cenegermin has recently been granted approval for use in Europe, thus the lack of published studies involving it. However, both substances promote epithelial healing to a reasonable degree. What would really be interesting is a study comparing these two agents in the treatment of neurotrophic keratopathy, in order to find out which substance is of greater benefit for these patients.

\section{REFERENCES}

1. Sacchetti M, Lambiase A. Diagnosis and management of neurotrophic keratitis. Clin Ophthalmol. 2014; 8: 571-579, doi: 10.2147/OPTH. S45921, indexed in Pubmed: 24672223.

2. Kauffmann T, Bodanowitz S, Hesse L, et al. Corneal reinnervation after photorefractive keratectomy and laser in situ keratomileusis: an in vivo study with a confocal videomicroscope. Ger J Ophthalmol. 1996; 5(6): 508-512, indexed in Pubmed: 9479547.

3. Wilson SE. Laser in situ keratomileusis-induced (presumed) neurotrophic epitheliopathy. Ophthalmology. 2001; 108(6): 1082-1087, indexed in Pubmed: 11382633.

4. Wilson SE, Ambrósio R. Laser in situ keratomileusis-induced neurotrophic epitheliopathy. Am J Ophthalmol. 2001; 132(3): 405-406, indexed in Pubmed: 11530056.

5. Netto MV, Mohan RR, Ambrósio R, et al. Gene therapy in the cornea. Prog Retin Eye Res. 2005; 24(5): 537-559, doi: 10.1016/j.preteyeres.2005.04.001, indexed in Pubmed: 15955719.

6. Lin X, Xu B, Sun Y, et al. Comparison of deep anterior lamellar keratoplasty and penetrating keratoplasty with respect to postoperative 
corneal sensitivity and tear film function. Graefes Arch Clin Exp Ophthalmol. 2014; 252(11): 1779-1787, doi: 10.1007/s00417-0142748-6, indexed in Pubmed: 25078353.

7. Wasilewski D, Mello GHR, Moreira H. Impact of collagen crosslinking on corneal sensitivity in keratoconus patients. Cornea. 2013; 32(7): 899-902, doi: 10.1097/ICO.0b013e31827978c8, indexed in Pubmed: 23263221.

8. Banerjee PJ, Chandra A, Sullivan PM, et al. Neurotrophic corneal ulceration after retinal detachment surgery with retinectomy and endolaser: a case series. JAMA Ophthalmol. 2014; 132(6): 750-752, doi: 10.1001/jamaophthalmol.2014.280, indexed in Pubmed: 24743924.

9. Tinley CG, Gray RH. Routine, single session, indirect laser for proliferative diabetic retinopathy. Eye (Lond). 2009; 23(9): 1819-1823, doi: 10.1038/eye.2008.394, indexed in Pubmed: 19136919.

10. Lockwood A, Hope-Ross M, Chell P. Neurotrophic keratopathy and diabetes mellitus. Eye (Lond). 2006; 20(7): 837-839, doi: 10.1038/ sj.eye.6702053, indexed in Pubmed: 16215544.

11. Mackie IA. Neuroparalytic keratitis. In: Fraunfelder F, Roy FH, Meyer SM. ed. Current ocular therapy. WB Saunders, Philadelphia, PA: 1995: 452-454.

12. Hersh PS, Rice BA, Baer JC, et al. Topical nonsteroidal agents and corneal wound healing. Arch Ophthalmol. 1990; 108(4): 577-583, indexed in Pubmed: 2322160.
13. Jeng BH, Dupps WJ. Autologous serum $50 \%$ eyedrops in the treatment of persistent corneal epithelial defects. Cornea. 2009; 28(10): 1104-1108, doi: 10.1097/ICO.0b013e3181a2a7f6, indexed in Pubmed: 19730088.

14. Turkoglu E, Celik E, Alagoz G. A comparison of the efficacy of autologous serum eye drops with amniotic membrane transplantation in neurotrophic keratitis. Semin Ophthalmol. 2014; 29(3): 119-126, doi: 10.3109/08820538.2013.768678, indexed in Pubmed: 23758337.

15. Bonini S, Rama P, Olzi D, etal. Neurotrophic keratitis. Eye (Lond). 2003; 17(8): 989-995, doi: 10.1038/sj.eye.6700616, indexed in Pubmed: 14631406.

16. Aifa A, Gueudry J, Portmann A, et al. Topical treatment with a new matrix therapy agent (RGTA) for the treatment of corneal neurotrophic ulcers. Invest Ophthalmol Vis Sci. 2012; 53(13): 8181-8185, doi: 10.1167/iovs.12-10476, indexed in Pubmed: 23150626.

17. http://ophthalmologytimes.modernmedicine.com/ophthalmologytimes/ news/cenegermin-receives-european-commission-marketing-authorization.

18. Faulkner WJ, Varley GA. Corneal diagnostic techniques. In: Krachmer $\mathrm{JH}$, Mannis MJ, Holland EJ. ed. Cornea. Elsevier/Mosby, Philadelphia 2005: 229-235.

19. Aslanides IM, Selimis VD, Bessis NV, et al. A pharmacological modification of pain and epithelial healing in contemporary transepithelial all-surface laser ablation (ASLA). Clin Ophthalmol. 2015; 9: 685-690, doi: 10.2147/OPTH.S81061, indexed in Pubmed: 25931809. 